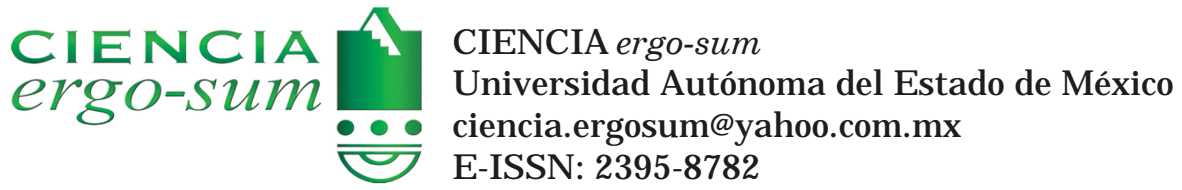

\title{
Migración intermunicipal permanente de la Zona Metropolitana del Valle de México 2010-2015
}

Pimienta Lastra, Rodrigo; Toscana Aparicio, Alejandra

Migración intermunicipal permanente de la Zona Metropolitana del Valle de México 2010-2015

CIENCIA ergo-sum, vol. 26, núm. 3, noviembre 2019-febrero 2020|e59

Universidad Autónoma del Estado de México, México

Esta obra está bajo una Licencia Creative Commons Atribución-NoComercial-SinDerivar 4.0 Internacional.

Pimienta Lastra, R. y Toscana Aparicio, A. (2019). Migración intermunicipal permanente dela Zona Metropolitana del Valle de México 2010-2015. CIE NCIA ergo-sum, 26(3). https://doi.org/10.30878/ces.v26n3a2 


\title{
Migración intermunicipal permanente de la Zona Metropolitana del Valle de México 2010-2015
}

Permanent Inter-municipal Migration of the Metropolitan Zone of the Valle de Mexico

Rodrigo Pimienta Lastra

Universidad Autónoma Metropolitana-Xochimilco, México

plrd6334@correo.xoc.uam.mx

Recepción: 12 de abril de 2018

Aprobación: 09 de julio de 2018

Alejandra Toscana Aparicio

Universidad Autónoma Metropolitana-Xochimilco, México

atoscana@correo.xoc.uam.mx

\section{Resumen}

Se estudian los movimientos migratorios intermunicipales de los 76 municipios y alcaldías que forman la Zona Metropolitana del Valle de México (ZMVM). Partiendo de los planteamientos de Lee, los axiomas propuestos por Pimienta y los datos de la Encuesta Intercensal 2015 del Instituto Nacional de Estadística y Geografía, los hallazgos muestran que los movimientos migratorios internos no han perdido vigencia, ya que entre 2010 y 2015 estas cifras fueron de 1046920 personas tan sólo en la ZMVM; los más importantes se tienen entre los municipios conurbados (41.3\%), seguidos de los movimientos de la Ciudad de México a la zona conurbada (20.7\%) y los menos de los municipios conurbados a la Ciudad de México (9.9\%).

Palabras Clave: migración interna, Zona Metropolitana del Valle de México, Encuesta Intercensal 2015.

\begin{abstract}
We study the intermunicipal migratory movements of the 76 municipalities and mayorships that form the Metropolitan Zone of the Valle de México (ZMVM). Based on the statements of Lee, the axioms proposed by Pimienta and the data from the Intercensal Survey 2015, we found the following: the internal migratory movements have not lost their validity as between 2010 and 2015, only in the ZMVM these figures were 1046920 people; the most important were between the conurbated municipalities (41.3\%), followed by the movement from Mexico City to the metropolitan area (20.7\%); the least important were from the municipalities surrounding Mexico City (9.9\%).
\end{abstract}

KEYWORDS: internal migration, Metropolitan Zone of the Valley of Mexico, Intercensal Survey 2015.

\section{INTRODUCCIÓN}

Con la publicación de la Encuesta Intercensal 2015 (INEGI, 2015a, 2015b, 2015c, 2015d) y su base de datos por parte del INEGI (Instituto Nacional de Estadística y Geografía) se abre un gran número de posibilidades para estudiar este fenómeno migratorio interno permanente del país en áreas geográficas relativamente pequeñas, esto es, a nivel municipal entre 2010 y 2015 en los 76 municipios que conforman la Zona Metropolitana del Valle de México (ZMVM). Si bien este tipo de estudios pudo iniciar desde que se publicaron las bases de datos de las muestras intercensales 2000 y 2010, no las habíamos tomando en cuenta porque nuestro trabajo en ese momento estaba centrado en el estudio y análisis de áreas de mayor tamaño: nacional y estatal. Ahora, con mayor experiencia tanto en el estudio del fenómeno migratorio como en el manejo de estas grandes bases de datos que proporciona el INEGI, hemos decidido incursionar en el análisis de la migración intermunicipal para lo cual hemos seleccionado la ZMVM.

Por esta razón, aquí retomamos el estudio de la migración interna desde un punto de vista descriptivo del fenómeno evitando lo que generalmente hace la academia: modelos sofisticados que poco entiende el común de las personas y poco aportan al estudio de un fenómeno tan complejo sin negar la importancia teórica y conceptual que tiene, pero que sería objeto de discusión en otro momento. 
En este trabajo, utilizando los datos de la Encuesta Intercensal 2015 realizada por el INEGI (2015), nos hemos concentrado en la descripción del fenómeno migratorio permanente por medio de algunos cuadros y gráficas de la ZMVM y sus 76 municipios que la conforman.

El artículo se encuentra dividido en seis apartados: Introducción, Planteamiento del problema, Antecedentes, Material y método de estudio, Análisis de resultados y Reflexiones finales, además de la parte correspondiente a las Referencias y los anexos estadísticos para ser confrontados con el desarrollo del texto y en los que podrá encontrarse información adicional.

\section{Planteamiento del problema}

A poco más de 125 años que Ravenstein sentó las bases de la reflexión científica del estudio de la migración al formular sus famosas Leyes de la migración (1889), donde expone los principios que regulan el fenómeno en Europa, poco se ha avanzado en los desarrollos teóricos del problema, pero sí mucho en la investigación aplicada. Lee en 1966 elabora una propuesta novedosa después de hacer una revisión de los autores que considera más importantes hasta ese momento, así como de los diversos enfoques que han abordo el estudio del fenómeno,.

Lee (1966) establece que la decisión y el proceso de migrar se pueden resumir en cuatro conceptos:

a) Factores asociados al lugar de origen

b) Factores asociados al lugar de destino

c) Obstáculos intervinientes

d) Factores personales

Sobre los dos primeros, Lee establece que hay factores positivos, negativos y neutros tanto en las áreas de atracción como de rechazo que influyen en la decisión del migrante potencial. El tercero refiere a la percepción que tiene el migrante sobre los problemas que se le pueden presentar antes, durante y al final de su desplazamiento. El cuarto está dirigido a la personalidad del migrante potencial y a la racionalidad con la cual el sujeto avalúa la decisión de migrar el ciclo de vida por el que atraviesa.

El conjunto de estos elementos definen las corrientes y contracorrientes tanto en las zonas de atracción como de rechazo, en este caso entre los municipios de la ZMVM, así como el volumen y la importancia de cada una de ellas.

Lee hace un señalamiento importante en su artículo: menciona que los estudios sobre el tema se han enfocado sobre todo a describir las características del migrante y que se han hecho pocas referencias sobre su volumen, por lo que propone diversos aspectos a considerar sobre esta variable; agrega que si la migración es una consecuencia de los factores positivos y negativos de las regiones de origen y destino, su volumen dentro de un territorio determinado varía con el grado de la diversidad de las áreas incluidas en él, la cual es una hipótesis que se ajusta a nuestra investigación y a su contrastación con nuestros datos (Lee, 1966).

Arango (1985), en su interpretación de Ravenstein, presenta la siguiente postura ante las críticas recibidas por algunos autores a su propuesta:

Por supuesto, soy absolutamente consciente de que nuestras leyes de población, y las leyes económicas en general, no tienen la rigidez de las leyes de la física, ya que están siendo continuamente interferidas por la acción humana. Las corrientes de migración que fluirían naturalmente en una cierta dirección, principalmente por las características geográficas, pueden ser desviadas o detenidas por completo por disposiciones legislativas (Arango, 1985: 7-26).

Es indudable que a la fecha no se ha logrado integrar un cuerpo teórico consistente que pueda establecer las leyes que describan el comportamiento del fenómeno migratorio, en el sentido estricto de las palabras. Al estudio del fenómeno se han incorporado diferentes áreas del conocimiento de las ciencias sociales en especial y de las 
formales como la matemática y la estadística, a las cuales les han estado sumando una gran cantidad de conceptos, tipologías y clasificaciones que resulta en un vocabulario cada vez más amplio en su abordaje.

En México el estudio de la migración interna es un fenómeno que había perdido vigencia después del auge que tuvieron las investigaciones que privilegiaron los grandes desplazamientos del campo a la ciudad, situación que ha ido cambiando en la primera década de este siglo. En los diversos eventos académicos que se realizan cada año sobre población pueden encontrarse mesas dedicadas al estudio de la migración interna, aunque esto se ha reflejado poco en la producción científica del país sobre el tema.

Partiendo de los planteamientos de Ravenstein y Lee, así como de los señalamientos de Arango, en este artículo en particular establece que el estudio de la migración tiene cuatro conceptos o axiomas básicos para su estudio (Pimienta, 2016):
a) Un lugar de origen
b) Un lugar de destino
c) El cruce de límites preestablecidos entre el origen y el destino
d) Una delimitación temporal

La migración no es una variable que se capta en las encuestas; por el contrario, es una variable que se construye a partir de los dos primeros axiomas y se define para cada investigación en particular considerando los dos últimos.

Partiendo de lo expuesto, establecemos que para considerar como migratorio el desplazamiento una persona debe abandonar su municipio de residencia habitual dentro de la ZMVM para irse a vivir en forma relativamente permanente a algún otro de la misma región, es decir, debe existir el cruce de alguno de los límites municipales dentro de esta zona. En este caso, tal movimiento debió darse entre 2010 y 2015. La delimitación temporal de nuestro planteamiento se la otorga la característica de permanencia y el tipo de fuente de información utilizada para el estudio: la Encuesta Intercensal 2015 realizada por el INEGI (2015d) en las variables municipio de origen en 2010 y de residencia en 2015, de las cuales se hablará más adelante. Finalmente, al utilizar los conceptos descritos pueden identificarse las zonas de atracción, equilibrio y rechazo de la población.

A partir de lo anterior, esta investigación con base en los datos de la Encuesta Intercensal 2015 hace estimaciones del volumen total de la migración interna permanente entre los municipios que integran la Zona Metropolitana del Valle de México, así como de cada uno de ellos con el fin de avaluar su importancia y establecer las corrientes principales que los alimentan.

\section{Antecedentes}

La Zona Metropolitana del Valle de México es el área geográfica más poblada del país y una de las 10 más pobladas del mundo. Está ubicada en el centro de la República Mexicana y la integran 76 municipios de tres de los estados de la región centro: 16 de la Ciudad de México, uno de Hidalgo y 59 del Estado de México. En 2015 albergó 17.5\% de la población del país (20 892724 de personas, casi la quinta parte). De esta cantidad de personas, 56.7\% (11 854 629) pertenece al Estado de México, 42.7\% (8 918 653) a la Ciudad de México y 0.6\% (119442) a Hidalgo (cálculos propios con base en datos de la Encuesta Intercensal 2015; INEGI, 2015d).

Es indudable que una de las principales causas de esta gran concentración humana inició desde hace varias décadas cuando comenzaron los grandes desplazamientos del campo a la Ciudad de México, que han sido muy estudiados por diversos autores, y después a los municipios que se encuentran en su entorno. Esta zona es un mosaico de nativos de prácticamente todas las entidades federativas que conforman la nación mexicana. En 2010 los estados que forman parte de esta zona sumaron en conjunto 7950774 inmigrantes permanentes, 1784571 de la Ciudad de México, 5726168 del Estado de México y 440035 de Hidalgo, que es 39\% de la migración total permanente interestatal del país en este periodo (Pimienta y Vera, 2015). 
Entre 2000 y 2010 estos estados recibieron 377395 personas; 326757 llegaron al Estado de México y 155796 al de Hidalgo, mientras que la Ciudad de México perdió 105 158, de ellas es probable que un número importante se haya desplazado al Estado de México y a Hidalgo (cálculos propios con base en Pimienta y Vera, 2005 y Pimienta y Vera, 2015).

Aparejada a la gran concentración urbana de la población al interior de esta zona, su dinámica migratoria intermunicipal se ha ido acentuando; por esta razón, en el artículo el estudio del fenómeno migratorio permanente será en el nivel municipal de agregación entre 2010 y 2015. Los movimientos poblacionales de esta zona pueden calificarse de muy intensos, ya que en el periodo mencionado se estima que estos flujos fueron de poco más de un millón de personas (1 046 920); el mayor número de ellos se presentó entre los municipios conurbados que rodean a la Ciudad de México, seguidos de los que ocurren entre los municipios (delegaciones) de la Ciudad de México. [1]

En el nivel de agregación que proponemos para el estudio prácticamente no hay trabajos de investigación realizados debido a que las pocas referencias encontradas son muy generales y están basados en algunos datos publicados por el INEGI.

\section{Materia y MÉtodo de eStudio}

Entre el 2 y el 27 de marzo de 2015, marcando como periodo de referencia el 15 de marzo, el Instituto Nacional de Estadística y Geografía (INEGI) elaboró la Encuesta Intercensal 2015, que contó con una muestra de 6133 946 viviendas de las que se obtuvo información de 22692265 personas a través de 126 variables de las características de la población y sus viviendas. Esta encuesta es probabilística con representatividad a nivel municipal y de localidades de cincuenta mil y más habitantes (INEGI, 2015). El diseño muestral que se utilizó es estratificado por conglomerados en una sola etapa de selección; las unidades primarias de muestreo (UPM) corresponden a agrupaciones de manzanas, cuya conformación buscó que todas las UPM tuvieran tamaños similares en términos de viviendas particulares habitadas (INEGI, 2015b).

El INEGI (2015) menciona en sus documentos que la encuesta permite obtener estimaciones de calidad de los totales de viviendas particulares habitadas y de la población residente para los dominios de estudio y análisis: la totalidad del país, sus 32 entidades federativas, los 2457 municipios y delegaciones del Distrito Federal y cada una de las localidades de cincuenta mil o más habitantes. De igual manera, se tiene la posibilidad de obtener estimadores[2] de proporciones, tasas y promedios de las variables de interés tanto para los niveles geográficos mencionados como para los dominios siguientes: entidad federativa para cinco tamaños de localidad predefinidos y cualquier agrupación geográfica del indicador, que por el tamaño de la muestra permita formar un nuevo dominio de estudio como zonas metropolitanas, regiones, etc. (INEGI, 2015b).

El INEGI pone a disposición del público en general la base de datos de la Encuesta Intercensal 2015 con la información de cada uno de los 32 estados del país. De cada estado se tienen dos archivos: el de personas y el de viviendas (INEGI, 2015d). Los módulos de personas de los estados de Hidalgo, Estado de México y Ciudad de México se integraron en un primer archivo, del cual se seleccionaron los 76 municipios que forman la ZMVM con el fin de formar un segundo archivo que cumpliera con los propósitos de nuestra investigación (cuadro 1 y mapa 1).

De este último archivo de datos se seleccionaron cuatro variables para definir el proceso migratorio interno de la región: a) ENT, $b$ ) MUN, $c$ ) ENT_PAIS_RES10 y d) MUN_RES10.Como lugar de residencia se tomaron las variables ENT y MUN, estado y municipio de residencia en la República Mexicana en 2015, correspondientes a la entidad federativa y municipio de la entidad federativa donde se levantó el cuestionario. Las otras dos variables: ENT_PAIS_RES10 y MUN_RES10 identifican el estado de la República Mexicana o país de residencia hace cinco años, en marzo de 2010, y municipio o delegación de residencia en el mismo periodo; de este archivo se eliminaron a los que residían en otro estado de la República Mexicana o en otro país en 2010. De esta forma, con estas dos últimas variables se establece el lugar de origen, en nuestro caso municipio, al cual podemos denominar lugar de residencia en 2010. 
CUADRO 1

Claves asignadas por el INEGI a los municipios de la Zona Metropolitana del Valle de México

\begin{tabular}{|c|c|c|c|c|c|}
\hline Núm. Mapa & Nombre & Clave INEGI & Núm. mapa & Nombre & Clave INEGI \\
\hline \multicolumn{6}{|c|}{ Municipios (Delegaciones) de la Ciudad de México } \\
\hline 1 & Azcapotzalco & 9002 & 9 & Álvaro Obregón & 9010 \\
\hline 2 & Coyoacán & 9003 & 10 & Tláhuac & 9011 \\
\hline 3 & Cuajimalpa de Morelos & 9004 & 11 & Tlalpan & 9012 \\
\hline 4 & Gustavo A. Madero & 9005 & 12 & Xochimilco & 9013 \\
\hline 5 & Iztacalco & 9006 & 13 & Benito Juárez & 9014 \\
\hline 6 & Iztapalapa & 9007 & 14 & Cuauhtémoc & 9015 \\
\hline 7 & La Magdalena Contreras & 9008 & 15 & Miguel Hidalgo & 9016 \\
\hline 8 & Milpa Alta & 9009 & 16 & Venustiano Carranza & 9017 \\
\hline \multicolumn{6}{|c|}{ Municipios de los estados de Hidalgo y México } \\
\hline 17 & Tizayuca (Hgo.) & 13069 & 47 & Nezahualcóyotl & 15058 \\
\hline 18 & Acolman & 15002 & 48 & Nextlalpan & 15059 \\
\hline 19 & Amecameca & 15009 & 49 & Nicolás Romero & 15060 \\
\hline 20 & Apaxco & 15010 & 50 & Nopaltepec & 15061 \\
\hline 21 & Atenco & 15011 & 51 & Otumba & 15065 \\
\hline 22 & Atizapán de Zaragoza & 15013 & 52 & Ozumba & 15068 \\
\hline 23 & Atlautla & 15015 & 53 & Papalotla & 15069 \\
\hline 24 & Axapusco & 15016 & 54 & $\mathrm{LaPaz}$ & 15070 \\
\hline 25 & Ayapango & 15017 & 55 & San Martín de las Pirámides & 15075 \\
\hline 26 & Coacalco de Berriozábal & 15020 & 56 & Tecámac & 15081 \\
\hline 27 & Cocotitlán & 15022 & 57 & Temamatla & 15083 \\
\hline 28 & Coyotepec & 15023 & 58 & Temascalapa & 15084 \\
\hline 29 & Cuautitlán & 15024 & 59 & Tenango del Aire & 15089 \\
\hline 30 & Chalco & 15025 & 60 & Teoloyucan & 15091 \\
\hline 31 & Chiautla & 15028 & 61 & Teotihuacán & 15092 \\
\hline 32 & Chicoloapan & 15029 & 62 & Tepetlaoxtoc & 15093 \\
\hline 33 & Chiconcuac & 15030 & 63 & Tepetlixpa & 15094 \\
\hline 34 & Chimalhuacán & 15031 & 64 & Tepotzotlán & 15095 \\
\hline 35 & Ecatepec de Morelos & 15033 & 65 & Tequixquiac & 15096 \\
\hline 36 & Ecatzingo & 15034 & 66 & Texcoco & 15099 \\
\hline 37 & Huehuetoca & 15035 & 67 & Tezoyuca & 15100 \\
\hline 38 & Hueypoxtla & 15036 & 68 & Tlalmanalco & 15103 \\
\hline 39 & Huixquilucan & 15037 & 69 & Tlalnepantla de Baz & 15104 \\
\hline 40 & Isidro Fabela & 15038 & 70 & Tultepec & 15108 \\
\hline 41 & Ixtapaluca & 15039 & 71 & Tultitlán & 15109 \\
\hline 42 & Jaltenco/Tonanitla & $15044 / 15125$ & 72 & Villa del Carbón & 15112 \\
\hline 43 & Jilotzingo & 15046 & 73 & Zumpango & 15120 \\
\hline 44 & Juchitepec & 15050 & 74 & Cuautitlán Izcalli & 15121 \\
\hline 45 & Melchor Ocampo & 15053 & 75 & Valle de Chalco Solidaridad & 15122 \\
\hline 46 & Naucalpan de Juárez & 15057 & 76 & Tonanitla & \\
\hline
\end{tabular}

Fuente: elaboración propia con datos del INEGI (Encuesta Intercensal 2015).

Nota: el municipio de Tonatitla (15125) se forma en 2003 con la división del municipio de Jaltenco (15044).

Para la realización de los mapas, en este trabajo ambos municipios serán considerados como uno solo con el fin de mantener la comparabilidad entre los años de estudio. 


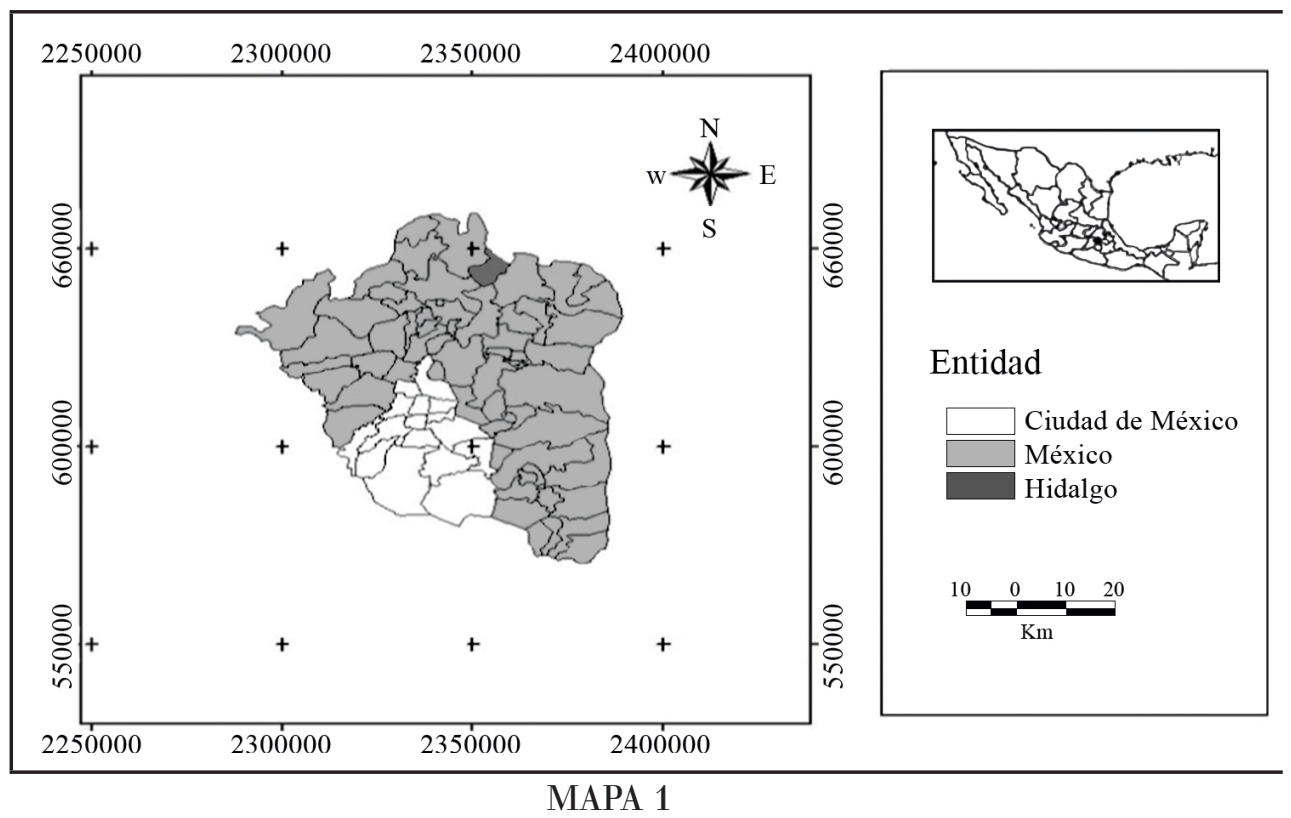

Delegaciones y municipios de la Zona Metropolitana de la Ciudad de México Fuente: elaboración propia con base en INEGI (2015).

Antes de iniciar el análisis de los datos es importante resaltar que en este estudio de la migración interna permanente planteada se utilizan los conceptos de lugar de residencia permanente en 2010 y lugar de residencia habitual en 2015, ambos casos en el ámbito municipal.

Para el proceso de los datos de las variables mencionadas se emplearon los paquetes computacionales MapInfo para la elaboración de la cartografía, Spss para la obtención de cuadros e indicadores y Excel para la elaboración de cuadros y gráficas.

\section{Análisis de resultados}

La Zona Metropolitana del Valle de México se ubica en el centro de la República Mexicana y está integrada por 76 municipios: 16 de la Ciudad de México, uno del estado de Hidalgo y 59 del de Estado de México. Esto nos lleva a tener que analizar una matriz de sus movimientos migratorios entre 2010 y 2015 de 76 renglones por 76 columnas. Para facilitar el análisis e interpretación de estos resultados, la matriz de entradas y salidas de población la dividiremos en cuatro submatrices: una que contenga sólo el intercambio migratorio entre los municipios (delegaciones) de la Ciudad de México, otra con los desplazamientos de la Ciudad de México hacia los municipios conurbados que la circundan, una más con los movimientos de estos últimos hacia la Ciudad de México y una cuarta con el intercambio migratorio entre los municipios conurbados que rodean la Ciudad de México (cuadros del 2 al 5 en el Anexo).

En el cuadro 2 (Anexo) se presenta la primera submatriz, donde podemos observar que entre 2010 y 2015 cambiaron su municipio de residencia dentro del área de la Ciudad de México 293879 personas, que es el segundo grupo en orden de importancia por su magnitud de movimientos migratorios permanentes en el periodo de estudio en la ZMVM. De ahí sobresalen, como municipios (delegaciones) que expulsan población, los de Iztapalapa con $14.6 \%$, Coyoacán $11.7 \%$, Benito Juárez 9.7\% y Cuauhtémoc 9\%, que representa $44.9 \%$ de la migración total de la zona, y en número de personas 132 019. Los principales sitios de atracción de migrantes fueron Benito Juárez con 13.7\%, Coyoacán 10.9\% e Iztapalapa $10.7 \%$ para un total de $31.3 \%$, que representa 92999 cambios de residencia. 


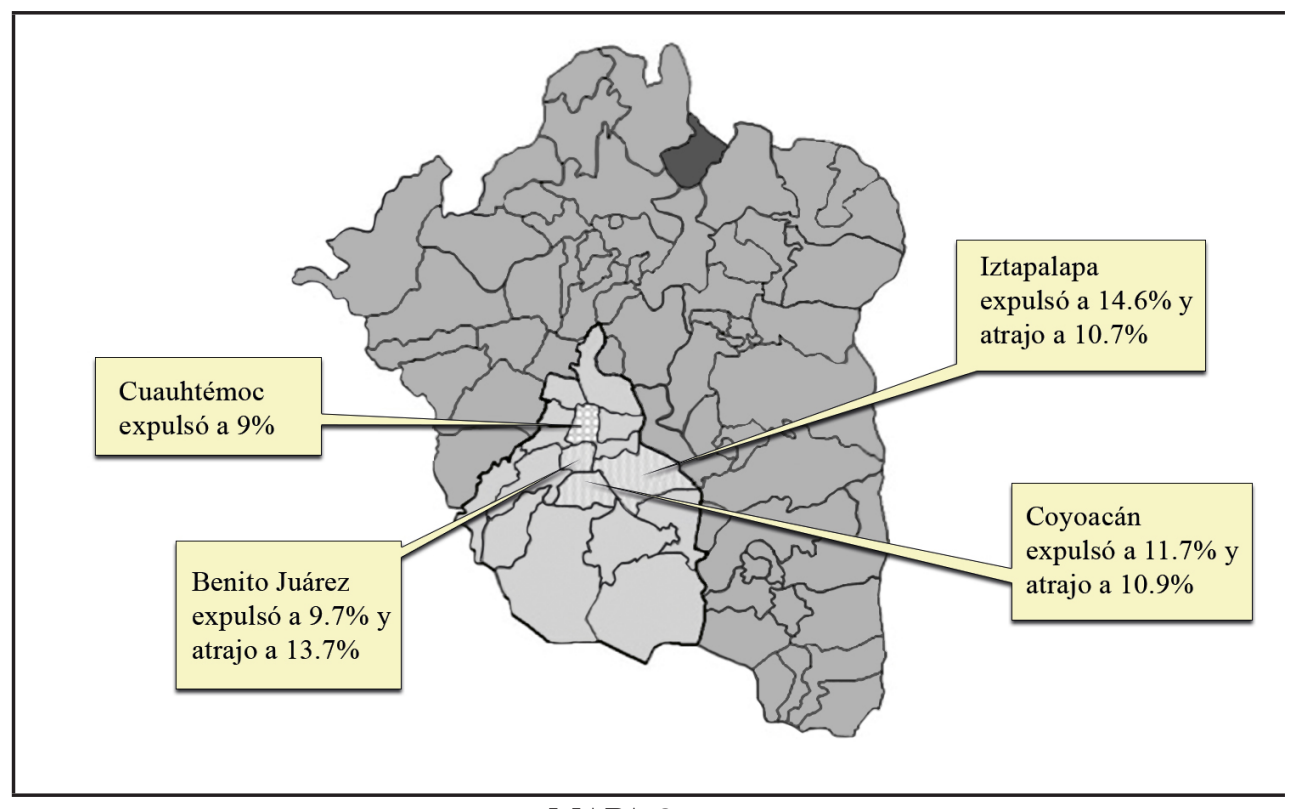

MAPA 2

Principales delegaciones expulsoras y receptoras de población Fuente: elaboración propia con base en INEGI (2015b).

En la segunda submatriz están los desplazamientos de la Ciudad de México hacia los municipios conurbados que la rodean, terceros en importancia por su magnitud: 217021 . En esta zona destacan como municipios de expulsión Iztapalapa con 25.1\% y Gustavo A. Madero con 21.7\%; le siguen en orden de importancia Azcapotzalco 8.3\%, Venustiano Carranza 6.2\% y Benito Juárez 6.1\%, que hacen un total de 158737 cambios de residencia. En este caso los municipios de atracción fueron Tecámac con 13.5\%, Ecatepec de Morelos 8.6\%, Chalco 8.4\%, Tlalnepantla de Baz 6.7\%, Nezahualcóyotl 6.3\%, Ixtapaluca 5.6\% y Valle de Chalco Solidaridad 5.1\%, que da un total de 60221 personas (cuadro 3 del Anexo y mapa 3).

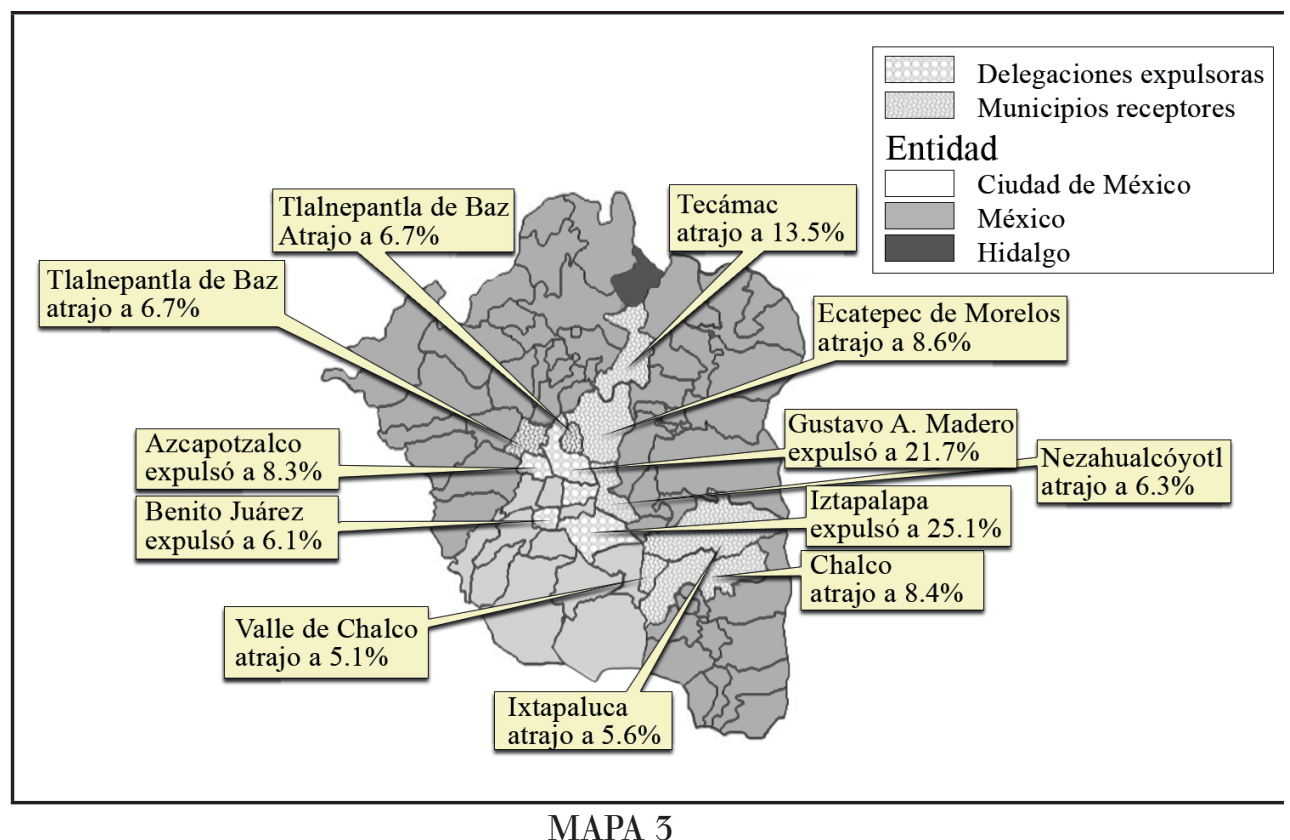

Principales delegaciones expulsoras de población a municipios y municipios receptores Fuente: elaboración propia con base en INEGI (2015b). 
En la tercera submatriz tenemos el cuarto grupo de desplazamientos en orden de importancia: los movimientos migratorios que tuvieron lugar de los municipios que rodean la Ciudad de México hacia ella, donde 103862 personas cambiaron su lugar de residencia. De esta zona sobresalen como municipios de expulsión Ecatepec de Morelos con 20.9\%, Nezahualcóyotl 19\%, Ixtapaluca y Naucalpan de Juárez 8.5\% cada uno y Tlalnepantla de Baz $6.1 \%$ que genera un total de 65306 desplazamientos. Como municipios de atracción de esta población tenemos Gustavo A. Madero con 19.5\%, Iztapalapa 17\%, Cuauhtémoc 11.2\%, Miguel Hidalgo 8\% y Azcapotzalco 7.9\% que representa 66082 movimientos migratorios (anexo cuadro 4).

En la cuarta submatriz se ubica en número de movimientos migratorios más importantes de la ZMVM los que se llevan a cabo entre los municipios conurbados ubicados alrededor de la Ciudad de México en el periodo de estudio; al respecto, 432158 personas cambiaron su lugar de residencia en esta zona. Los municipios más importantes de expulsión de la zona son Ecatepec de Morelos con 18.9\% y Nezahualcóyotl 12.8\%, le siguen en segundo lugar Naucalpan de Juárez 8.8\% y Tlalnepantla de Baz 7.7\% y en tercer sitio Tultitlán con 5\%, Cuautitlán Izcalli 4.3\%, Ixtapaluca 3.5\% y Chimalhuacán 3.2\%, que en total suman 277640 cambios de residencia. Como municipios de atracción de población se tienen como lugar principal Tecámac con 10.3\%, seguido muy de lejos por Zumpango 5.9\%, Chimalhuacán 5.5\%, Tultitlán 5.4\%, Huehuetoca e Ixtapaluca 4.6\% cada uno, Ecatepec de Morelos 4.3\%, Chalco 4.1\% y Atizapán de Zaragoza 3.9\%, que hacen un total de 209484 desplazamientos migratorios (cuadro 5 del Anexo).

\section{REFLEXIONES FINALES}

El estudio de la migración interna no pierde vigencia, puesto que se mantiene como un fenómeno demográfico de suma importancia al seguir configurando la distribución espacial de la población en nuestro país. Entre 2000 y 2010 los movimientos migratorios interestatales fueron de 2969 975. En un periodo de cinco años, entre 2010 y 2015, tan sólo en la ZMVM estos desplazamientos alcanzaron la cifra de 1046 920; el más importante se dio entre los municipios conurbados (432 158, 41.3\%), seguido por los que acontecieron entre los municipios de la Ciudad de México (293 879, 28.1\%).

En tercer lugar están los movimiento de la Ciudad de México a los municipios conurbados $(217$ 021, 20.7\%). Los menos importantes fueron los desplazamientos de los municipios conurbados a la Ciudad de México (103 $862,9.9 \%)$.

Con esto se puede establecer que se tienen dos zonas de fuerte migración que aunque se encuentran próximas geográficamente en términos migratorios tienen su propia dinámica; por un lado, la dinámica interna de la Ciudad de México y la de los municipios que se encuentran a su alrededor y, por otro, el intercambio migratorio que se da entre estas dos zonas, aunque con menor intensidad del que sucede dentro de ellas. Las primeras dos zonas se pueden clasificar como de muy alta y alta migración y las dos restantes como de baja y muy baja migración.

Analizando este tipo de movimientos y al relacionarlos con otras variables como costo de la vivienda, ingreso familiar, niveles educativos y actividad laboral de las personas, se puede encontrar una buena parte de la explicación: a) el relativo equilibrio en población que ha mantenido durante algunos años la Ciudad de México y b) la dinámica migratoria que han desarrollado los municipios que se encuentran a su alrededor.

\section{AnÁlisis PROSPECTIVo}

La importancia del estudio de la migración en las zonas metropolitanas del país seguirá vigente dada la tendencia de crecimiento de las ciudades y sus zonas metropolitanas. Las políticas urbanas, habitacional, de ordenamiento del territorio, de planeación y dotación de equipamiento e infraestructura deberán tener en cuenta los movimientos migratorios internos permanentes con la finalidad de lograr el bienestar de la población si se atienden los municipios receptores de población. 


\section{REFERENCIAS}

Arango, J. (1985). Las "Leyes de las migraciones" de E. G. Ravenstein, cien años después. Reis: Revista Española de Investigaciones Sociológicas, 32, 7-26.

INEGI (Instituto Nacional de Estadística y Geografía). (2015). Encuesta Intercensal 2015. Características generales. Aguascalientes: Instituto Nacional de Estadística y Geografía.

INEGI (Instituto Nacional de Estadística y Geografía). (2015a). Encuesta Intercensal 2015. Principales resultados. Aguascalientes: Instituto Nacional de Estadística y Geografía.

INEGI (Instituto Nacional de Estadística y Geografía). (2015b). Encuesta Intercensal 2015. Sintesis metodológica y conceptual. México: Instituto Nacional de Estadística y Geografía.

INEGI (Instituto Nacional de Estadística y Geografía) (2015c). Encuesta Intercensal 2015. Principales resultados de la encuesta intercensal 2015: Estados Unidos Mexicanos. Aguascalientes: Instituto Nacional de Estadística y Geografía.

INEGI (Instituto Nacional de Estadística y Geografía). (2015d). Encuesta Intercensal 2015. Base de datos, módulos: características de las personas y características de la vivienda: Estados Unidos Mexicanos. Aguascalientes: Instituto Nacional de Estadística y Geografía.

Lee, E. S. (1966). A theory of migration. Demography, 3(1), 47-57.

Pimienta, R. y Vera, M. (2005). Dinámica migratoria interestatal de la República Mexicana, Zinacantepec: El Colegio Mexiquense.

Pimienta, R. y Vera, M. (2015). Migración interna en México. Datos de la muestra censal de 2010. México: Universidad Autónoma Metropolitana Unidad Xochimilco, Universidad Autónoma del Estado de México.

Pimienta, R. (2016). Los mapas de la migración interna en México. México: Universidad Autónoma Metropolitana Unidad Xochimilco.

Ravenstein, E. G. (1889). The laws of migration. Journal of the Royal Statistical Society, 52(2), 241-305.

\section{Notas}

[1] La Ciudad de México tomó este nombre como consecuencia de la reforma constitucional del 29 de enero de 2016, de ahí que las delegaciones pasarán a ser municipios o su equivalente.

[2] Un estimador es un indicador (estadístico) calculado de las observaciones hechas sobre una variable a partir de una muestra, en este caso probabilística, lo que permite hacer inferencias sobre toda la población. 
Anexo

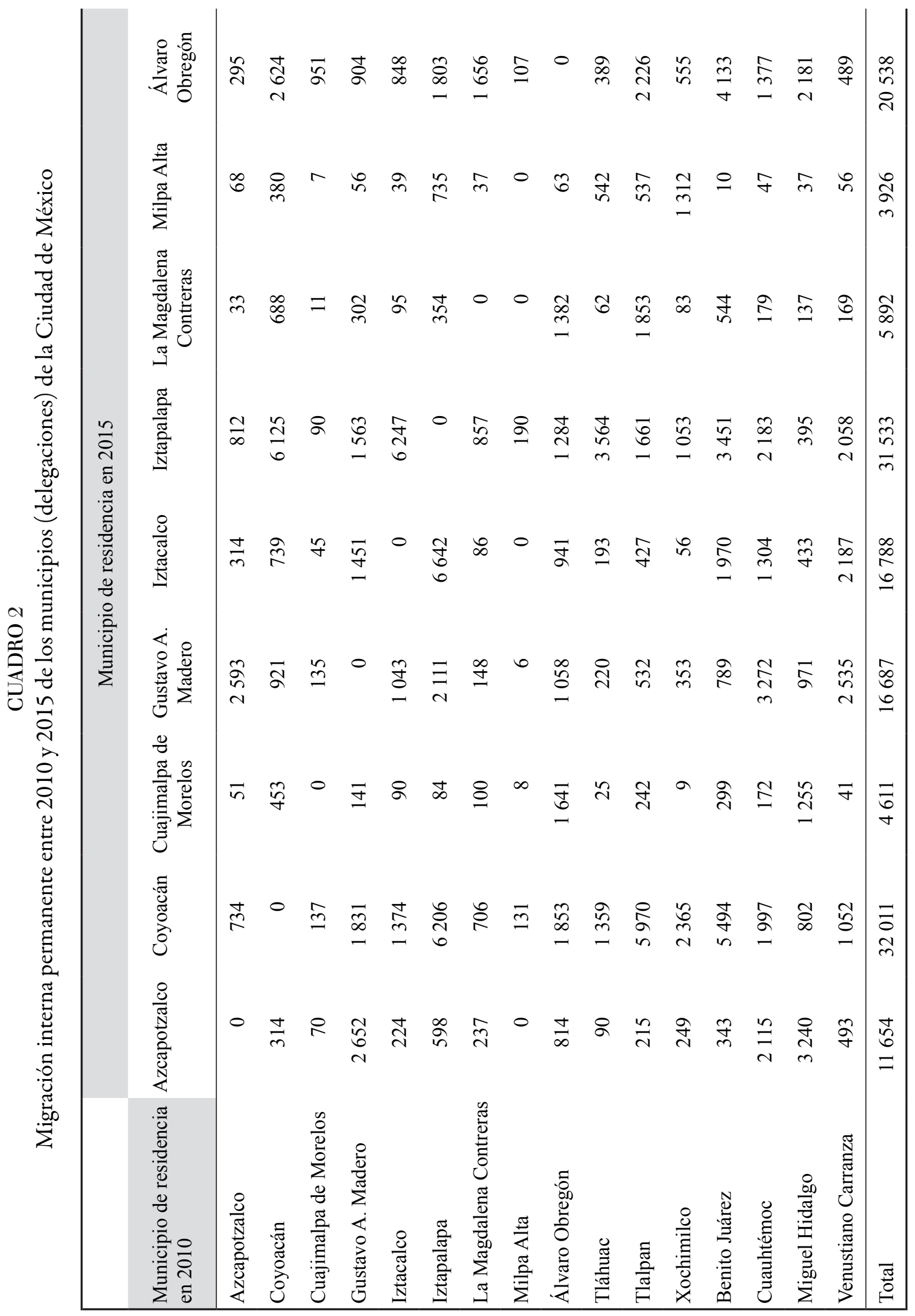




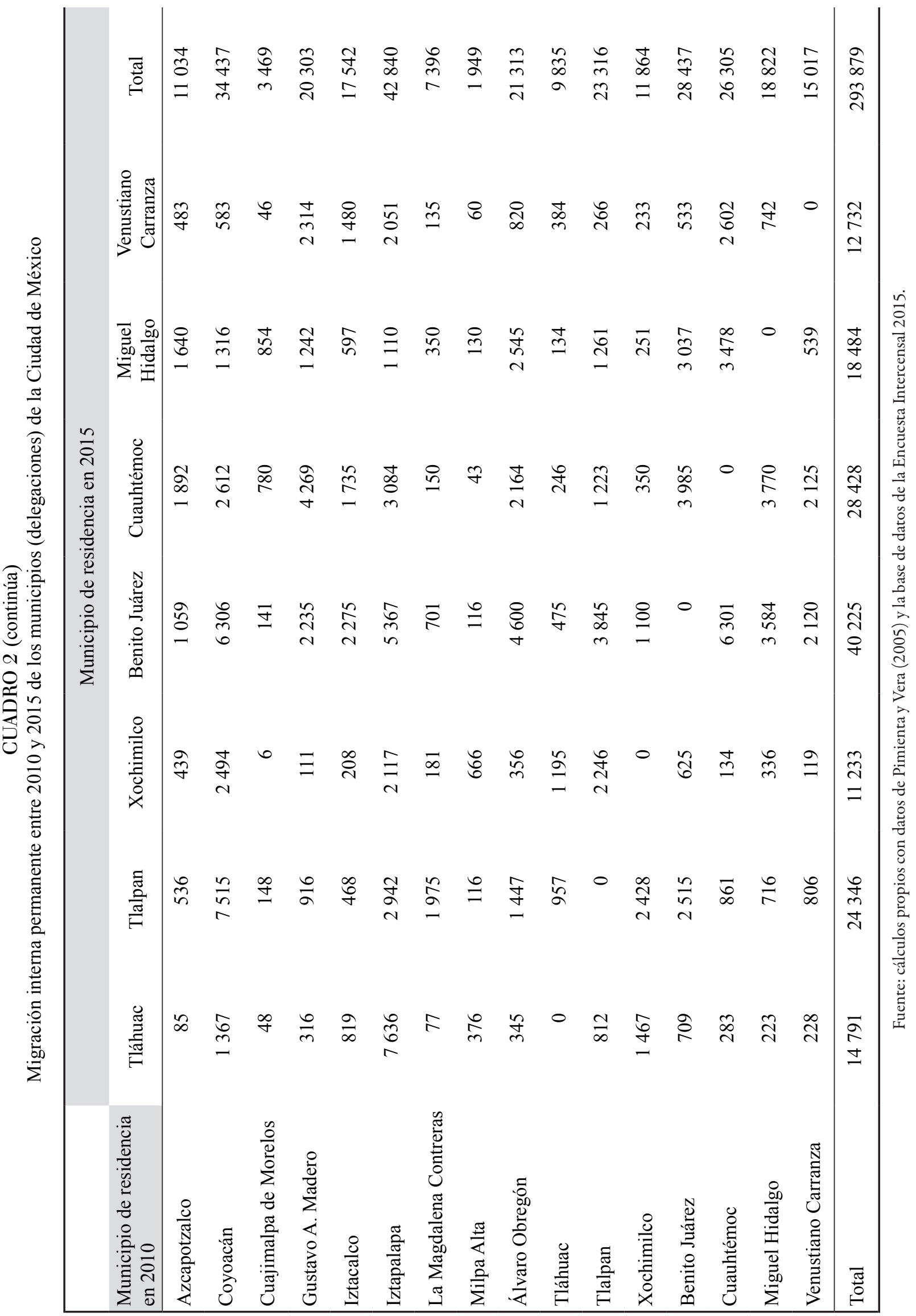




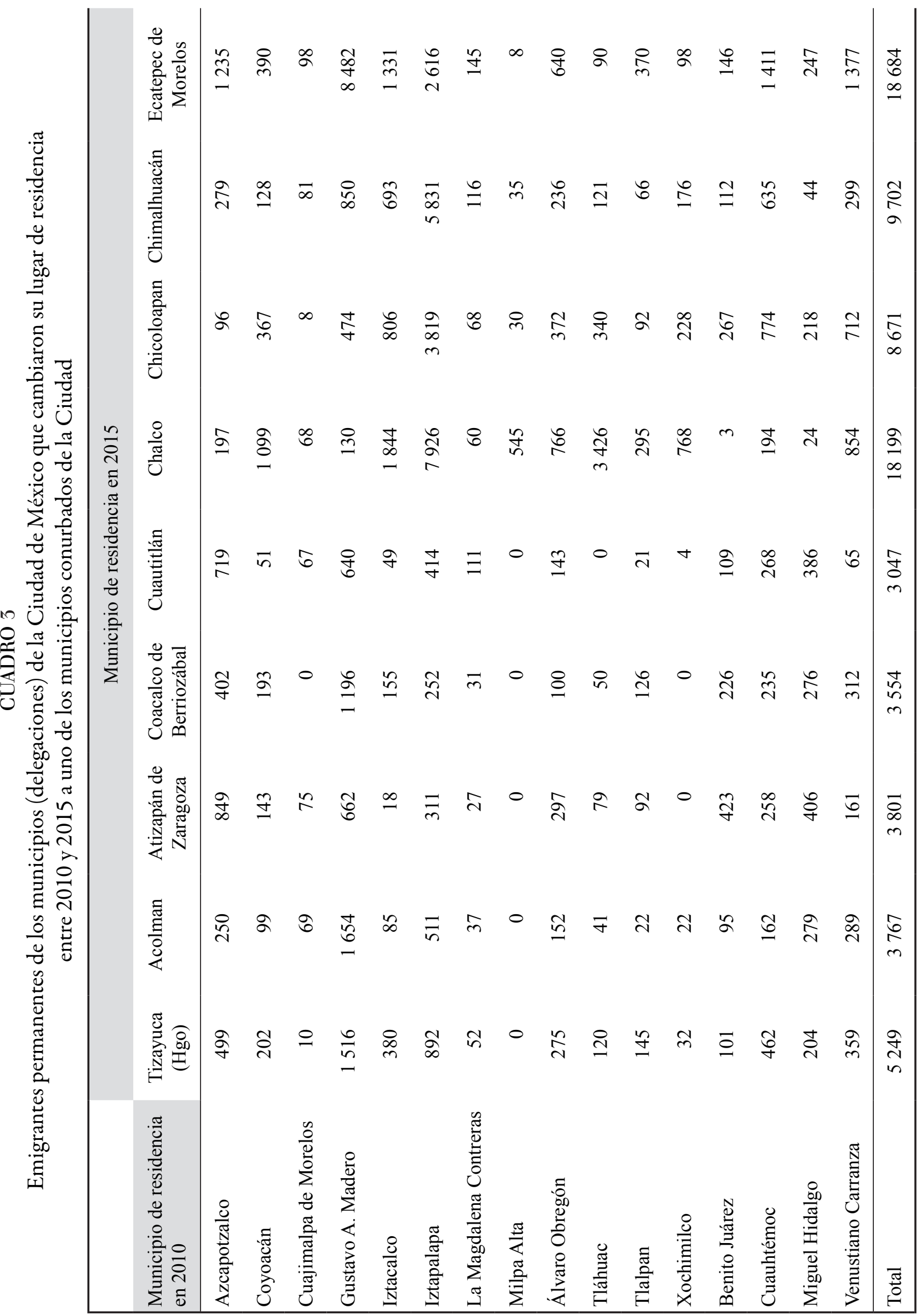




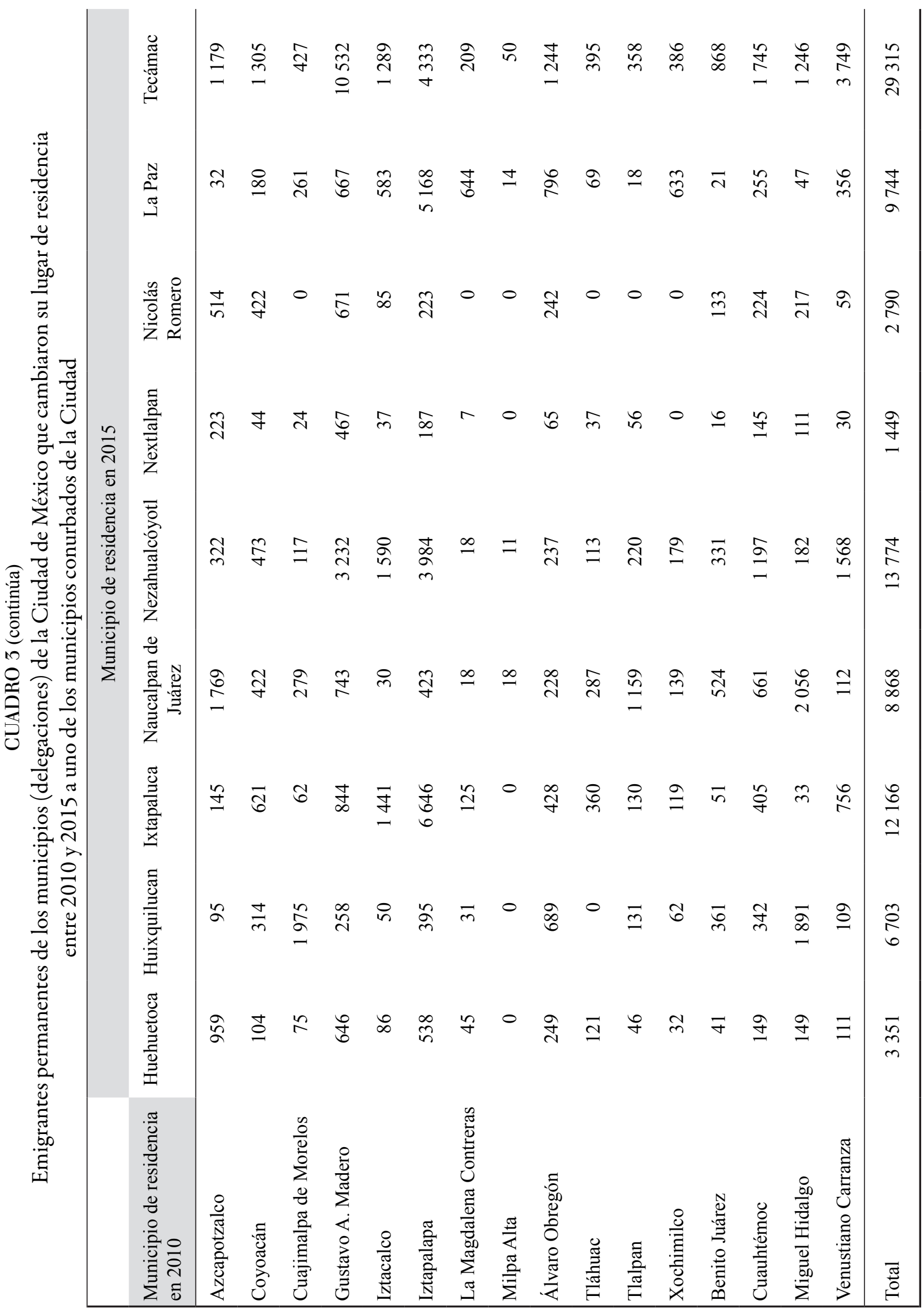




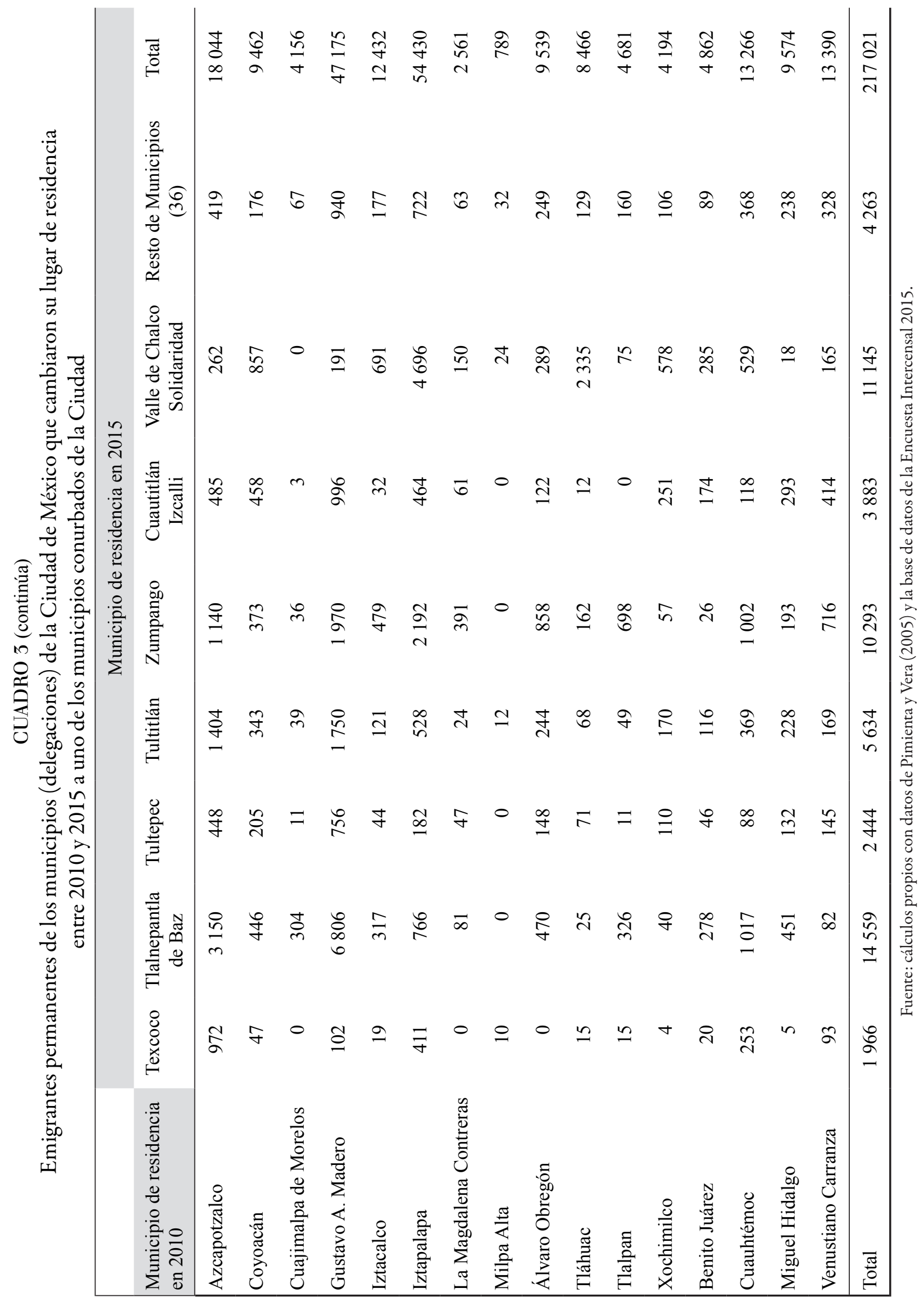




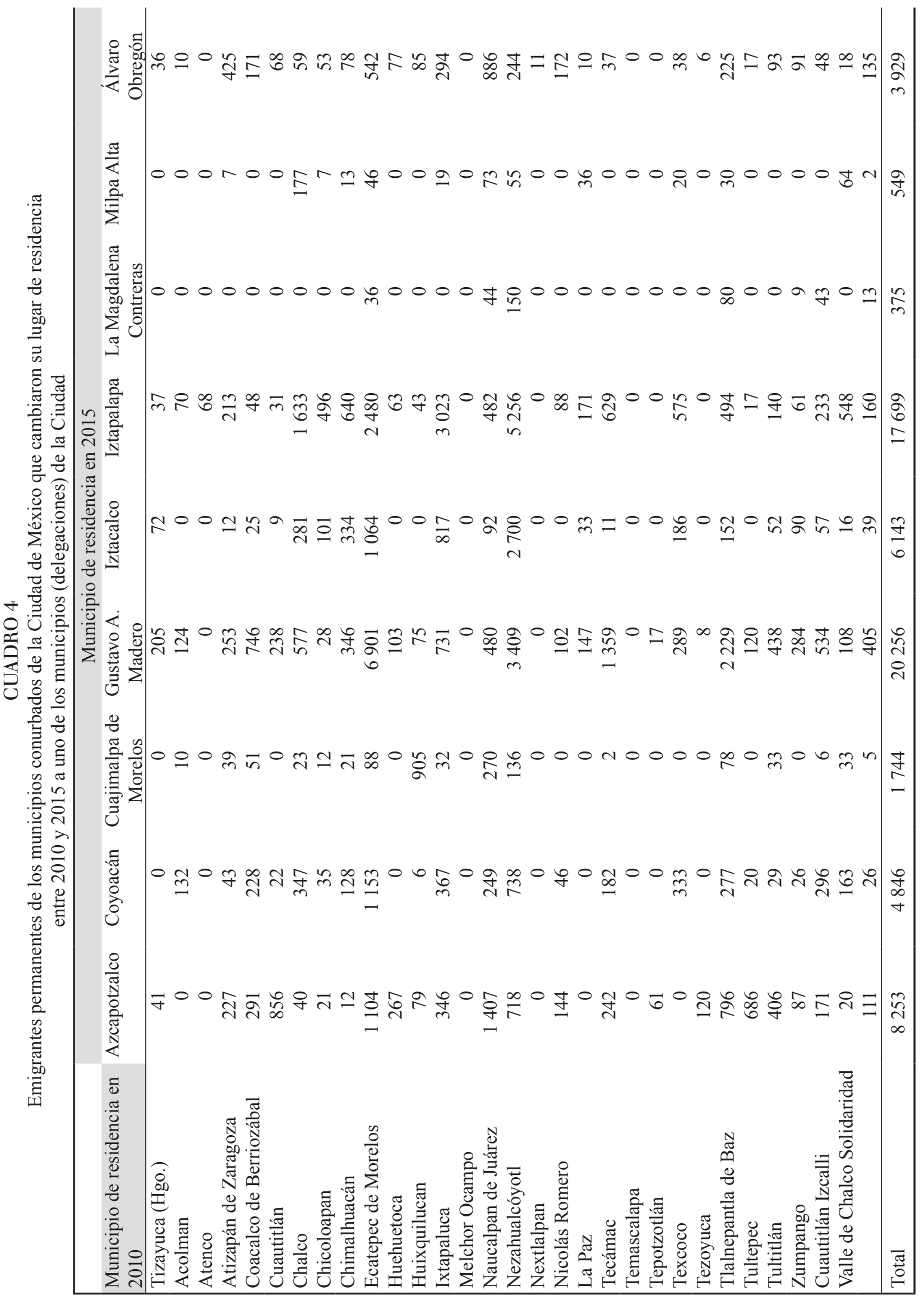




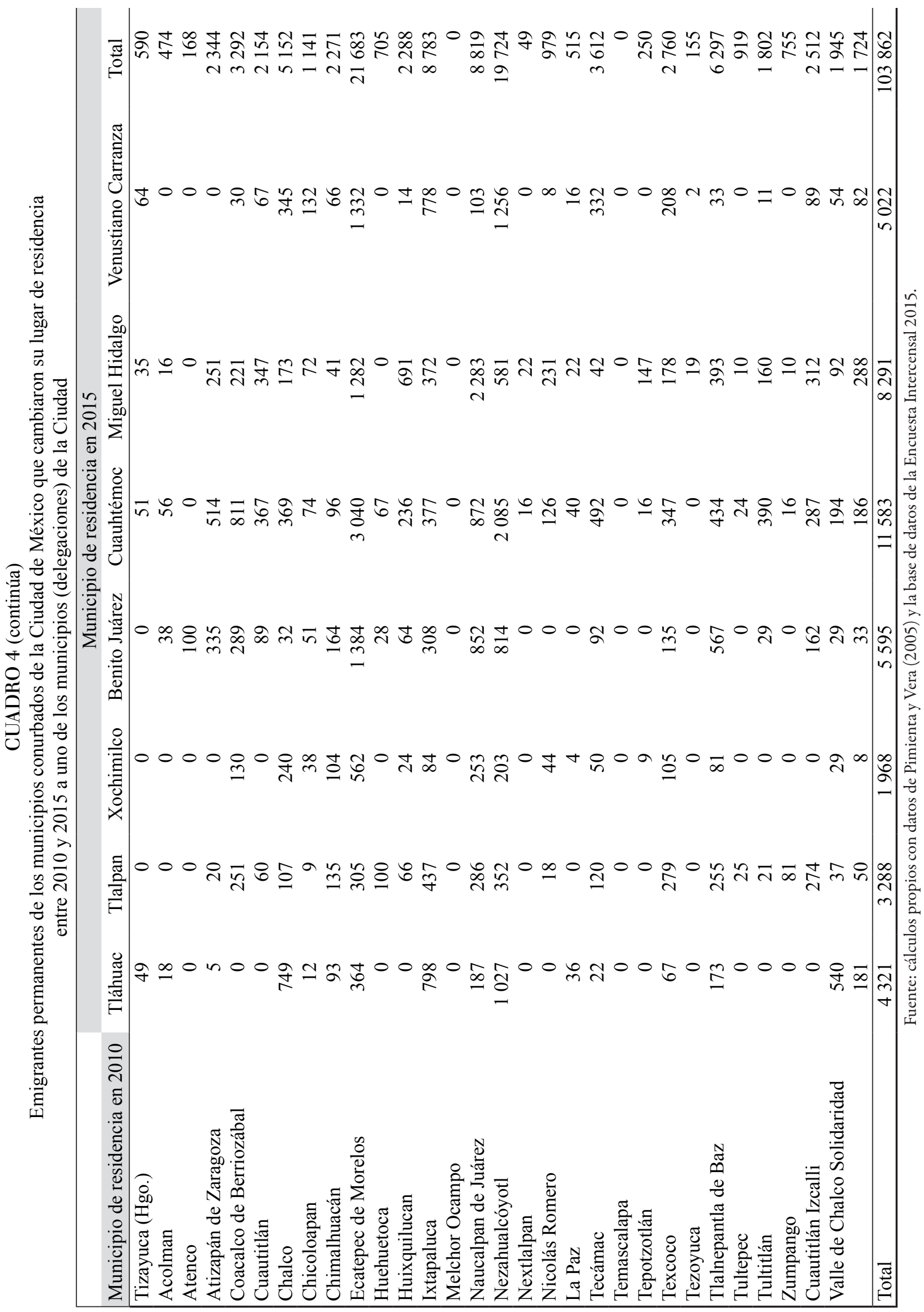




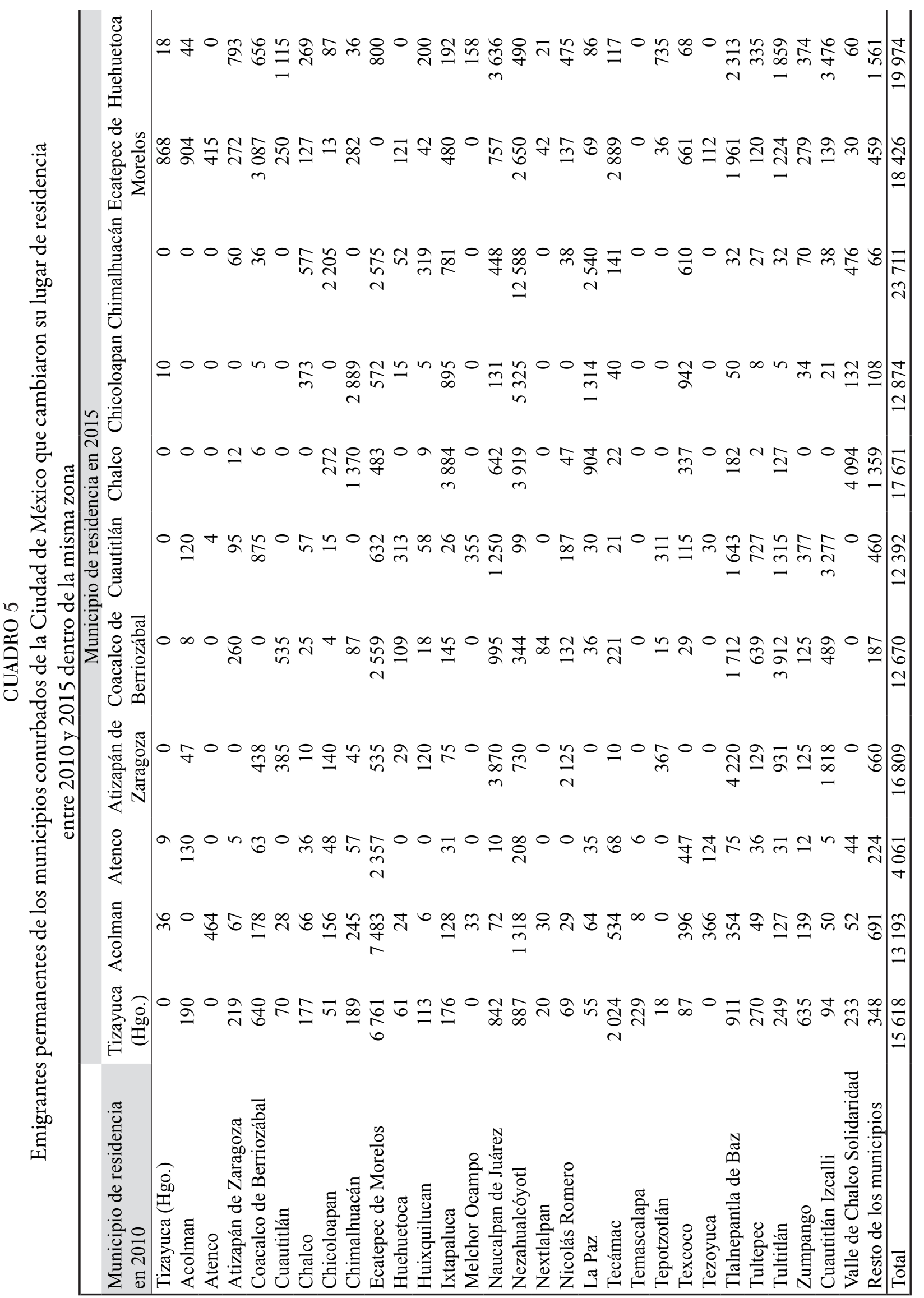




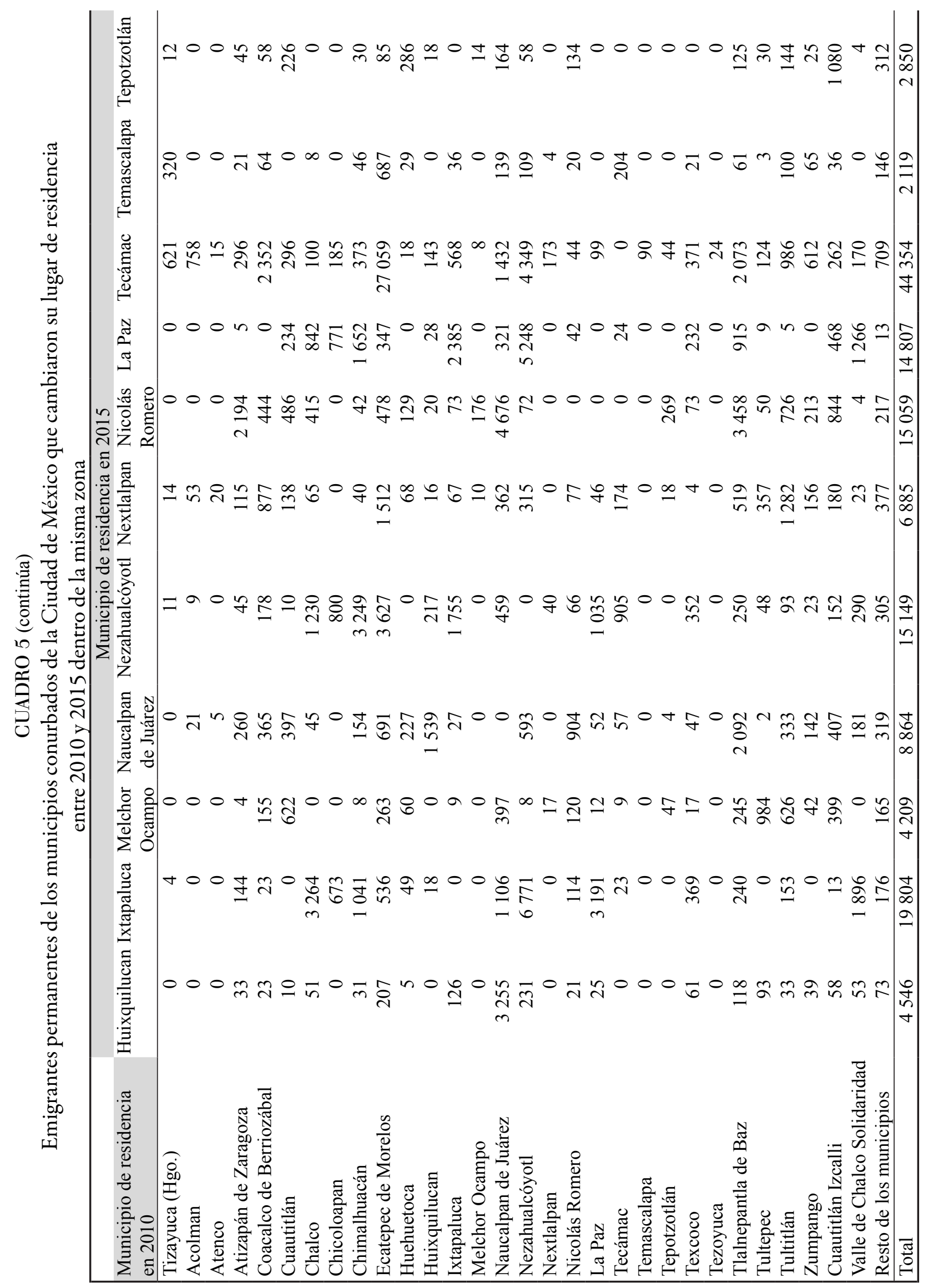




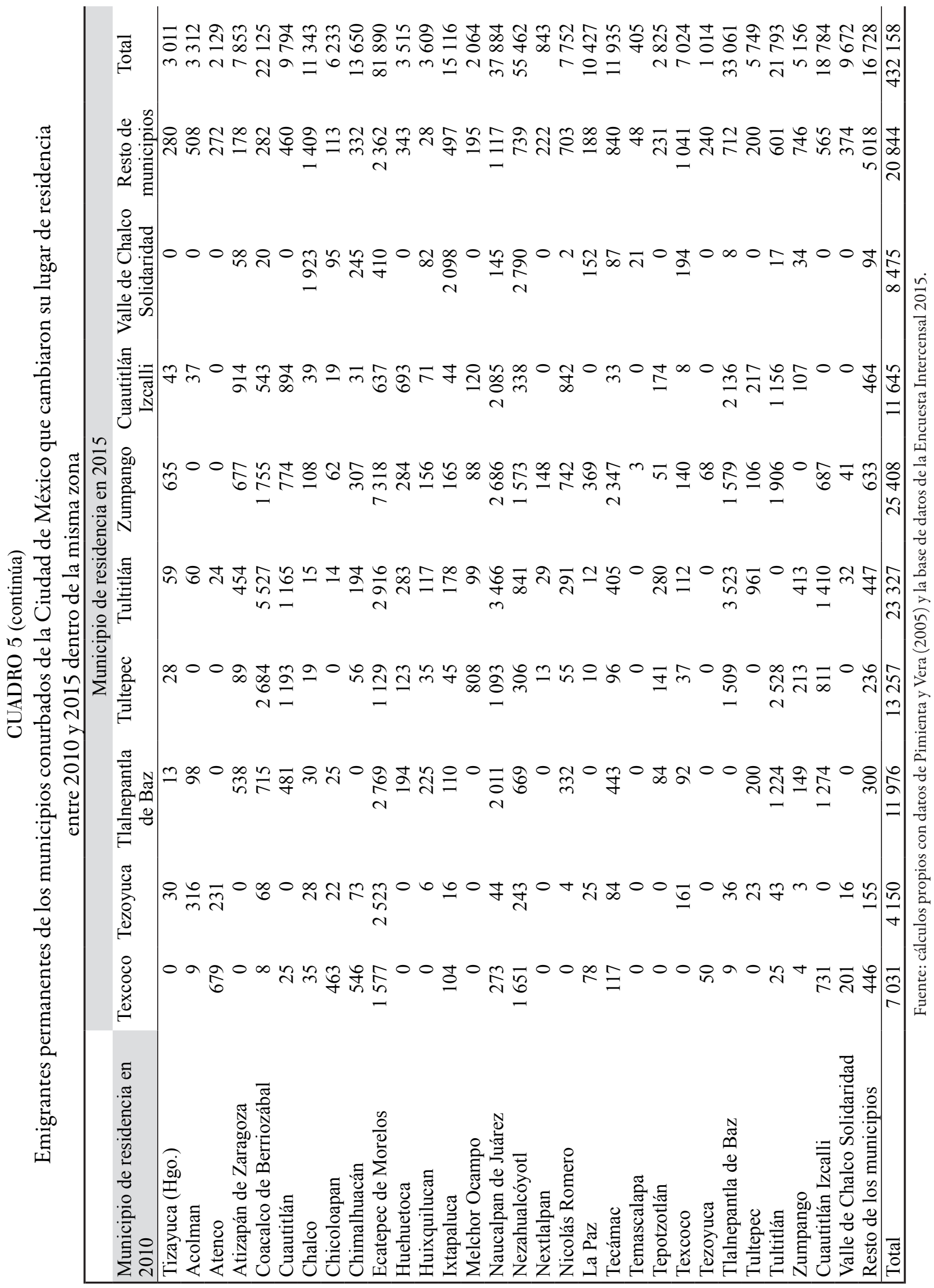

CC BY-NC-ND 